$\mathrm{AB} 0485$

INTRAVENOUS HIGH DOSE GLUCOCORTICOIDS CAUSE PROLONGATION OF QT INTERVAL IN CONNECTIVE TISSUE DISEASE PATIENTS EXCEPT ANTI-RO POSITIVE SUBGROUP

Tomáś Soukup ${ }^{1}$, Jan Toms ${ }^{2}$, Radek Pudil ${ }^{2}$, Jakub Simka ${ }^{2}$, Lenka Pollak ${ }^{3}$, Nikola Jurikova ${ }^{3}$, Martin Dosedel ${ }^{3}$, Jiri Vlcek ${ }^{3} .{ }^{1}$ Charles University, Faculty of Medicine and University Hospital in Hradec Kralove, 2nd Department of Internal Medicine-Gastroenterology, Division of Rheumatology, Hradec Králové, Czech Republic; ${ }^{2}$ Charles University, Faculty of Medicine and University Hospital in Hradec Kralove, Hradec Králové, Czech Republic; ${ }^{3}$ Charles University, Faculty of Pharmacy in Hradec Kralove, Hradec Králové, Czech Republic

Background: Recent literature indicates that anti-Ro antibodies may be associated with prolongation of corrected QT (QTC) interval in the adult patients with connective tissue diseases. Moreover, glucocorticoids (frequently used in the therapy) are known to probably induce electrocardiographic changes including prolongation of QT interval. Prolongation of QTC interval is a risk factor for malignant ventricular arrhythmias. We hypothetised that patients with anti-Ro positivity have a higher risk of QT prolongation especially in the case of high dose intravenous glucocorticoids (IVGC) treatment. Objectives: The aim of the study was to analyse risk of QTC interval prolongation in anti-Ro and anti-La positive patients before and after high dose IVGC treatment in patients with connective tissue diseases.

Methods: We performed a retrospective study of 115 patients $(21$ males), mean age of $48 \pm 14.7$ years, range 19-78 years with connective tissue disease. Anti-Ro antibodies were examined in all patients before IVGC treatment. The patients were given $5 \times 1000 \mathrm{mg}$ methyprednisolon i.v. during five consecutive days. ECG recording was performed at baseline and after IVGC. The QT intervals were measured in each of 12-lead standard ECGs from two consecutive cycles. The QT intervals were measured from the onset of QRS complex to the end of the $T$ wave by the means of a tangential method. Fridericia formula was used to obtain heart rate-corrected values for QT intervals.

Results: Comparing patients with anti-Ro positivity $(n=39 ; 33.9 \%)$ and anti Ro-negativity $(n=76,66.1 \%)$, we found insignificant difference in QTc interval; QTcRo+ $=417.2 \pm 28.4 \mathrm{~ms}$ and QTcRo- $=420,8 \pm 26.4 \mathrm{~ms} \quad(P=0.51)$, respectively. Admission of IVGC showed significant prolongation of QTC in all patients, mean QTc1 before treatment was $399.4 \pm 26.2 \mathrm{~ms}$, and QTc2 after therapy $412.7 \pm 27.2 \mathrm{~ms}, P=0.00021$. On the other hand, anti-Ro and anti-La positivity showed shorter QTc interval $391 \pm 18.7 \mathrm{~ms}$, to compare patients without these antibodies $\mathrm{QTC}=414.8 \pm 25.7 \mathrm{~ms}, \mathrm{P}=0.019$ after treatment.

Conclusion: IVGC treatment significantly prolongs QT interval. Prolongation of QT over $445 \mathrm{~ms}$ was found in nine patients, but ventricular arrythmias were not observed. The presence of anti-Ro and anti-La antibodies did not show QT prolongation. Therefore, shortening of QT/QTC iterval needs further confirmation. Motivated by this experience, we consider ECG monitoring and careful observation of patients during IVGC treatment.

Acknowledgement: Charles University research projects [PROGRES Q4015, PROGRES Q47]

Disclosure of Interests: None declared

DOI: 10.1136/annrheumdis-2019-eular.4253

\section{AB0486 LACK OF CONSISTENT STANDARD OF CARE IN SYSTEMIC LUPUS ERYTHEMATOSUS PATIENTS IN REAL WORLD}

Zahi Touma ${ }^{1}$, Ben Hoskin², Christian Atkinson ${ }^{2}$, David Bell ${ }^{2}$, Olivia Massey², Jennifer H. Lofland ${ }^{3}$, Pam Berry ${ }^{4}$, Chetan Karyekar ${ }^{3}$, Karen Costenbader ${ }^{5}$. ${ }^{1}$ University of Toronto, Toronto, Canada; ${ }^{2}$ Adelphi Real World, Cheshire, United Kingdom; ${ }^{3}$ Janssen Global Commercial Strategic Organisation, Horsham, United States of America; ${ }^{4}$ Janssen Global Services, LLC, Horsham, United States of America; ${ }^{5}$ Brigham and Women's Hospital, Boston, United States of America

Background: Current guidelines do not necessarily reflect current standard of care (SOC) or treatment patterns in SLE in real world.

Objectives: To describe SLE SOC in the EU5 and US and assess need for a more efficient and defined treatment pathway.

Methods: A cross-sectional study of 263 rheumatologists in the US and EU5. Data were collected from the Adelphi Real World 2015 Lupus Disease Specific Programme (DSP). Physicians were asked to complete patient record forms (PRFs) for the next 5 patients consulting with SLE; the same patients were asked to complete patient self-completion (PSC) forms describing how SLE affected them. PRFs collected data pertaining to the patient's diagnosis, disease history, current clinical outcomes, treatment and management history. PSCs focused on similar data collection and included patient reported outcome measures (PROs) to assess the humanistic burden. Chi-squared tests were conducted to ascertain significance.
Results: Data was extracted from 1376 PRFs, and 591 PSCs. $35.6 \%$ of patients were currently on their first line of therapy, $40.0 \%$ second line, and third line or more $24.4 \%$. Significant differences were observed between US and EU5 in current treatment classes for first line $(p<0.0001)$ and second line $(p=0.0007)$. Descriptive differences were observed in the third line.

In the US monotherapy is used more often first line with antimalarial (AM) (monotherapy EU5 $12.5 \%$ vs. US $19.3 \%$ ) and immunosuppressant (IM) (monotherapy EU5 $4.6 \%$ vs. US 11.1\%). Glucocorticoids (GCS) use is higher in the EU5 at $1^{\text {st }}$ line; both monotherapy (EU5 $13.7 \%$ vs. US $11.8 \%$ ) and combination (GCS+AM EU5 $28.3 \%$ vs. US $16.7 \%$ ). At second line, IM use (no bio) is higher in EU5 (EU5 64.9\% vs. US $56.7 \%$ ) and biologic use is higher in the US (EU5 $5.0 \%$ vs. US $8.3 \%$ ); GCS use is similar (EU5 $19.2 \%$ vs. US $19.0 \%$ ).

Globally, $84.4 \%$ of patients receiving GCS received it continuously with no significant differences observed between markets, EU5 $85.5 \%$ vs. US $81.9 \%$. A higher proportion of patients in the EU5 have been on GCS for 6 months or longer when compared to the US; EU5 $79.9 \%$ vs. US $72.5 \%$. Statistically significant differences were seen in the perception of GCS importance between markets $(p=0.0004)$ and concerns with taking GCS $(p=0.0140)$. A higher proportion of patients in the US regarded the use of GCS to at least be very important (very important/essential EU5 $65 \%$ vs. US $80.6 \%$ ). A higher proportion of patients in the US were concerned with the use of GCS (somewhat concerned/very concerned EU5 $56 \%$ vs. US $61.2 \%$ )

Conclusion: Significant differences in treatment approach between regions highlights the need for a better understanding of this disparity and a united approach to SLE treatment. Despite the high profile of risk factors linked to GCS use in SLE, it continues to be a continuously utilized by a large proportion of SLE patients and there is poor use of flare preventing agents such as antimalarials. Patients and physicians recognize the role of GCS in the management of SLE but also express concerns. Impact of an update to the SLE guidelines and their dissemination with better understanding of risk benefits of GCS vs. immunosuppressive and biologic therapy is warranted.

Disclosure of Interests: Zahi Touma Grant/research support from: GSK Canada, Consultant for: UBC, Pfizer, Janssen, Inc, Ben Hoskin Employee of: Adelphi, Christian Atkinson Employee of: Adelphi, David Bell Employee of: Adelphi, Olivia Massey Employee of: Adelphi Real World, Jennifer $\mathrm{H}$ Lofland Employee of: Janssen Global Commercial Strategic Organization, Pam Berry Shareholder of: GSK and Janssen Global Services, Chetan Karyekar Shareholder of: J\&J, Employee of: Janssen Scientific Affairs, LLC, Abbott, BMS, Novartis, Karen Costenbader: None declared DOI: 10.1136/annrheumdis-2019-eular.4392

\section{AB0487 HCQ COULD ACT ON SLE PATIENTS THROUGH THE MODULATING EXPRESSION OF IL-8 ALONG WITH S100 PROTEINS}

Risa Wakiya, Kiyo Ueeda, Shusaku Nakashima, Hiromi Shimada, Mai Mahmoud Fahmy Mansour, Mikiya Kato, Taichi Miyagi, Tomohiro Kameda, Hiroaki Dobashi. Kagawa University, Department of Internal Medicine, Division of Hematology, Rheumatology and Respiratory Medicine, Kagawa, Japan

Background: Some reports revealed that S100A8 and S100A9 proteins were associated with disease activity of lupus nephritis(LN) [1]. However there have been no reports about the mechanism of additional hydroxychloroquine (HCQ) treatment for systemic lupus erythematosus (SLE) patient with low disease activity.

Objectives: To clarify the mechanism of additional HCQ treatment for Japanese SLE patients with low disease activity.

Methods: All the 44 patients were enrolled in this study. These patients had been receiving additional oral HCQ sulfate continuously for at leas 3 months. These patients had no need for additional immunosuppressants including glucocorticoids, during this study because of their sustained low disease activity for 3 months prior to starting HCQ. Low disease activity was defined as SELENA-SLEDAI score of 8 or less with no activity in major organ systems. As conventional immunologica and clinical assessment for SLE disease activity were determined to examine the levels of complement (C3, C4, CH50), anti-dsDNA antibody, blood cell count, SELENA-SLEDAI and CLASI score. Serum levels of S100A8 and S100A9 reported as novel markers for SLE disease activity[1] were measured using ELISA (CircuLex ELISA Kit, MBL) at the time of $\mathrm{HCQ}$ administration as well as 3 months later. In addition, several expressions of cytokines (IFN- $\alpha$, IFN- $\gamma$, TNF- $\alpha$, IL-2, IL-4, IL-5, IL-6, IL-8, IL-10, IL-17, IL-1 $\alpha$, IL-1 $\beta$, IL-1ra, VEGF, GM-CSF, G-CSF, CCL2, CCL3, CCL4, CXCL) were measured by multiplex assay (Human Luminex assay, R\&D systems). 


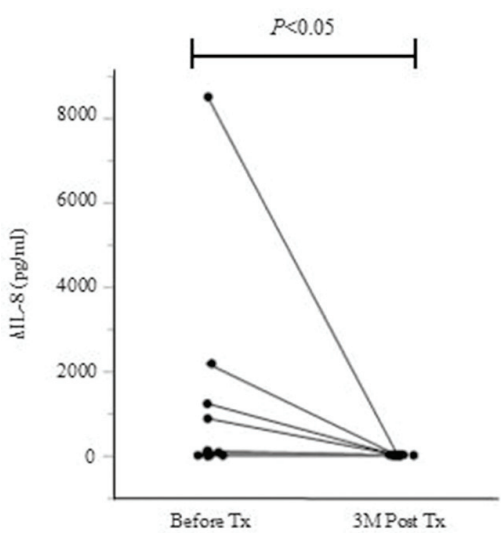

Figure 1 . Serum level of IL -8 decreased significantly 3 months after additional HCQ treatment.

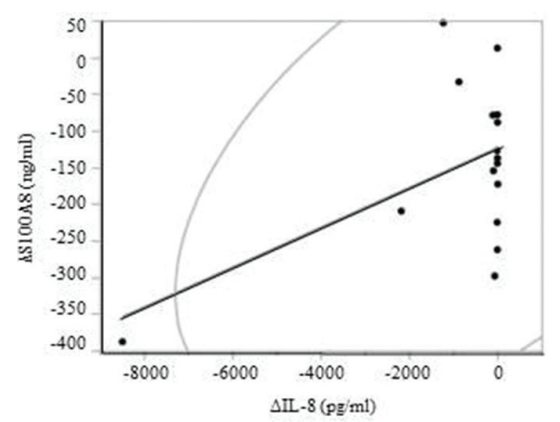

Figure 2. Correlation between the change of $I L-8$ and that of S100A\& protein $(r=0.51, \mathrm{p}=0.044)$.

Results: We enrolled 44 patients. Prednisolone dose during this study was fixed at the mean of $5.0 \pm 2.9 \mathrm{mg}$ per day. SELENA-SLEDAl scores, CLASI scores, anti-dsDNA antibody and serum levels of C3 improved significantly, and serum levels of S100A8 and S100A9 proteins decreased significantly 3 months after additional $\mathrm{HCQ}$ treatment. The changes in serum S100A8 and S100A9 levels in SLE patients with LN were more significantly higher than in those without $L N$.

Among these patients, the changes of serum cytokine expressions were measured in 18 patients. The expressions of IFNs were not detected in almost of all patients. Serum levels of TNF- $\alpha$, IL-6, IL-8, IL-1 $\beta$, IL-1ra, VEGF, CCL3 and CCL4 decreased significantly 3 months after additional $\mathrm{HCQ}$ treatment (Figure1).

Seven of 18 SLE patients had a history of LN. These patients have been in sustained complete remission(CR) of LN for over one year(mean duration of $\mathrm{CR}$ was $5.0 \pm 3.5$ years).

The reductive effect of additional HCQ treatment on serum S100A9, TNF$\alpha$, IL-6 and IL-8 levels was much more apparent in those with history of LN (LN: S100A9, $p=0.016$, TNF- $\alpha, p=0.016, \quad I L-6 \quad p=0.031, I L-8, p=0.031$; no LN: S100A9, $p=0.065$, TNF- $\alpha, p=0.083$, IL-6 $p=0.090, \quad \mathrm{LL}-8, p=0.557$ ). The changes of serum S100A8 and S100A9 levels were correlated with those of serum IL-8 and IL-1ra (Figure2).

Conclusion: $\mathrm{HCQ}$ could reduce the serum levels of $\mathrm{S} 100$ protein and several cytokines in SLE Japanese patients with low disease activity. Recently, some reports showed that novel biomarker including IL-8 or S100 proteins was correlated with severity of $\operatorname{LN}[1,2]$. Our findings suggest that $\mathrm{HCQ}$ treatment without any additional immunosuppressant could reduce the expression of serum $\mathrm{S} 100$ proteins, IL-8 and IL-1ra, which were considered to be associated with the improvement of renal and life prognosis. Our data also indicated that $\mathrm{S} 100$ protein is closely related to IL-8 or IL-1ra expression in SLE pathogenesis, especially in LN. Farther investigations are needed to more clarify the significance of $\mathrm{HCQ}$ treatment in SLE.

\section{REFERENCES}

[1] P Tantivitayakul, et al. Lupus. 2016(25):38-45.

[2] A El-shehaby, et al. J Clin Immunol. 2011(5):848-56.

\section{AB0488}

RITUXIMAB THERAPY IN LUPUS NEPHRITIS RESISTANT TO CONVENTIONAL THERAPY - A SINGLE CENTER EXPERIENCE (CASE SERIES)

Berna Yurttas, Bugra Han Egeli, Serdal Ugurlu. Istanbul University-Cerrahpasa, Cerrahpasa Medical Faculty, Department of Internal Medicine, Division of Rheumatology, Istanbul, Turkey

Background: Lupus nephritis $(\mathrm{LN})$ can be a cause of morbidity and mortality in a significant portion of patients. The treatment of lupus nephritis can be challenging in some patients who are resistant to conventional immunosuppressive treatment.

Objectives: Rituximab is a promising agent for treating resistant patients We evaluated the response of lupus nephritis patients who were treated with rituximab treatment in our clinic retrospectively.

Methods: We evaluated LN patients who were followed and treated with at least one course of rituximab in our clinic between 2013-2018. Remission was defined as proteinuria below $500 \mathrm{mg} /$ day. Also, we evaluated the reason behind the cessation of rituximab during the follow-up whether it is a side effect or lack of efficacy. A final 24-hour proteinuria level was also analyzed at the end of the follow-up.

Results: 33 patients $(20 \mathrm{~F}, 13 \mathrm{M})$ were treated with rituximab. Al patients had active lupus nephritis at initiation. Mean of disease duration was 6.7 years. Diagnosis was proven by renal biopsy, except two (Table). Patients received an average of 4.2 courses. All patients were treated with high dose steroids. The other medications, the patients were previously treated with, were Cyclophosphamide $(n=26)$, Azathioprine $(n=7)$, Mycophenolate mofetil $(n=17)$. Also one patient was treated with plasmapheresis due to thrombotic thrombocytopenic purpura. 2 patients were lost in follow-up. 13 patients $(39 \%)$ are still on rituximab. Six of them $(46 \%)$ still have active LN. Among 18 patients in whom rituximab therapy was terminated, 3 had inadequate response, 2 had severe infection (cellulitis, pneumonia), 1 had Rituximab-induced serum sickness, 1 had will of pregnancy. Treatment of 11 patients was terminated owing to remission of disease. All of the patients whom rituximab was terminated in disease remission did not flare, except one. The mean level of pretreatment proteinuria was $3356 \pm 2478 \mathrm{mg} /$ day. The mean level of proteinuria after the final course was $1025 \pm 982 \mathrm{mg} /$ day $(p<0,0001)$. Mean erythrocyte sedimentation rate (ESR) was reduced from 28,62 $\pm 22,95$ $\mathrm{mm} / \mathrm{h}$ to $14,97 \pm 12,32 \mathrm{~mm} / \mathrm{h} \quad(\mathrm{p}<0,005)$. While initial steroid dose was $24 \pm 19,3 \mathrm{mg} /$ day, steroid dose at last rituximab course was $7,84 \pm$ $6,13 \mathrm{mg} /$ day $(p<0,0001)$. None of the patients had a new organ involvement during therapy. End stage renal disease was developed in 3 patients. During the treatment 7 patients had infections, 2 had herpes zoster, 1 had cellulitis, 3 had pneumoniae and 1 had frequent upper respiratory infections.

Table. Histological characteristics of patients

\begin{tabular}{lc}
\hline RPS/ISN Classification & Number of patients \\
\hline Class II & 1 \\
Class III & 4 \\
Class IV & 11 \\
Class V & 7 \\
Class III+IV & 6 \\
Class IV+V & 2 \\
\hline
\end{tabular}

Conclusion: In lupus nephritis patients resistant to conventional therapy, rituximab can be tried as an alternative treatment choice. In our single center, most of the patients were class IV nephritis whom we observed the efficacy of rituximab. Generally, the side effects were acceptable. Rituximab can be a steroid sparing agent in these patients.

\section{REFERENCES}

[1] laccarino L, Bartoloni E, Carli L, Ceccarelli F, Conti F, De Vita S, Ferraccioli G, Galeazzi M, Gatto M, Gerli R, Govoni M, Gremese E, luliano A, Mansutti E, Moroni G, Mosca M, Nalli C, Naretto C, Padovan M, Palma L, Raffiotta F, Roccatello D, Tincani A, Valesini G, Zen M, Doria A. Efficacy and safety of off-label use of rituximab in refractory lupus: data from the Italian MulticentreRegistry. Clin Exp Rheumatol. 2015 Jul-Aug;33(4):44956 .

Disclosure of Interests: None declared DOI: 10.1136/annrheumdis-2019-eular.7284 